\title{
ANÁLISIS DE LA SECUENCIA DE VISITA A GRANADA DE LOS TURISTAS: DIFERENCIAS POR ORIGEN Y GRADO DE EXPERIENCIA CON EL DESTINO
}

\author{
ROCÍO MARTÍNEZ SUÁREZ \\ Departamento Comercialización e Investigación de Mercados, UNIVERSIDAD DE GRANADA, ESPAÑA \\ e-mail: rmartinez@ugr.es \\ JOSÉ ALBERTO CASTAÑEDA GARCÍA \\ Departamento Comercialización e Investigación de Mercados, UNIVERSIDAD DE GRANADA, ESPAÑA \\ e-mail: jalberto@ugr.es \\ MIGUEL ÁNGEL RODRÍGUEZ MOLINA \\ Departamento Comercialización e Investigación de Mercados, UNIVERSIDAD DE GRANADA, ESPAÑA \\ e-mail: rmolina@ugr.es \\ JULIO VENA OYA \\ Departamento Comercialización e Investigación de Mercados, UNIVERSIDAD DE GRANADA, ESPAÑA \\ e-mail: juliovena@correo.ugr.es
}

\section{RESUMEN}

El objetivo del trabajo es identificar la ruta típica de distintos conjuntos de turistas (nacionales vs. extranjeros; nóveles vs de repetición) en un destino cultural, como es Granada. Haciendo uso de la herramienta 'Cronología de Google', se obtiene la secuencia o ruta de visita seguida por más de 300 turistas. Tras adaptar y contextualizar los datos para este ámbito de estudio, se aplica el Alineamiento de Secuencias, metodología propia de la investigación en bioquímica. Como resultado se obtiene que el lugar de origen del turista y el número de visitas previas condicionan el comportamiento espacio-temporal de los turistas. Así, los turistas de repetición preferirán no visitar lugares con mucha aglomeración de visitantes, excepto si son turistas no nacionales, en cuyo caso deciden volver a visitar el principal atractivo de la ciudad. Este trabajo pretende poner de manifiesto la utilidad del alineamiento de secuencias dentro de la investigación turística.

Palabras clave: Alineamiento de Secuencias, Secuencia de visita, Lugar de origen, Visitas previas, Granada, Historial de ubicaciones Google, GPS

\section{Tourists' sequence of visit to Granada : differences by origin and experience with the destination}

\begin{abstract}
The objective of the study is to identify the typical route of different sets of tourists (national vs. foreign, new vs. repeat) in a cultural destination, such as Granada. Using 'Google Location History', we get visit sequence followed by more than 300 tourists. After adapting and contextualizing the data for this field of study, Sequence Alignment is applied, a methodology used in biochemical research. The result is that the original place of tourists and the number of previous visits determine the spatial-temporal behavior of tourists. Then, repeat tourists prefer not to visit places with a lot of visitors, except if they are non-national tourists, in which case they decide to revisit the main attraction of the city. This work aims to highlight the usefulness of sequence alignment in tourism research.
\end{abstract}

Keywords: Sequences alignment, Visit sequence, Original place, Repeat visit, Granada, Google Location History, GPS

Clasificacion JEL: Z32

Artículo recibido el 15 de noviembre de 2018 y aceptado el 5 de enero de 2019

Artículo disponible en versión electrónica en la página www.revista-eea.net 


\section{INTRODUCCIÓN}

España es uno de los países que recibe anualmente un mayor número de visitantes. Según los datos proporcionados por el Ministerio de Energía, Turismo y Agenda Digital, en el año 2017 visitaron el país más de 82 millones de extranjeros, lo que supone un incremento del $8,9 \%$ respecto al año anterior. Estos datos reflejan que 2017 fue el año con más recepción de turistas internacionales, lo que suma ya cinco años consecutivos batiendo este record. Estos datos hacen que España supere por primera vez a Estados Unidos y ocupe ya la segunda posición en el ranking de países que atraen a un mayor número de visitantes, solo superado por Francia.

Con respecto al gasto que realizaron estos turistas, se superaron los 87.000 millones de euros, alcanzando un nuevo record, y que supone un incremento del $12,4 \%$ respecto a 2016 , lo que equivale a un gasto medio por turista de $1.061 €$ y un gasto medio diario de $137 €$ (INE, 2017).

Estos datos ponen de nuevo de manifiesto la importancia que tiene para España el sector turístico, haciendo que sea imprescindible su estudio y que se siga trabajando en la mejora con el objetivo de ocupar la primera posición del ranking mundial en recepción de turistas extranjeros.

Todos los datos anteriores refuerzan la idea de que tanto los destinos turísticos como las empresas que en ellos operan se preocupen por ofrecer a sus turistas experiencias de calidad que aumenten tanto su satisfacción como su intención de recomendar el destino o incluso de volver en un futuro (Geva y Goldman, 1991; Knutson y Beck, 2004).

Estudios recientes han demostrado que la experiencia que tienen los turistas en un destino está conectada con la forma en la que el turista visita el destino, es decir, el recorrido que realice el turista va a determinar en gran medida su experiencia con el destino (Pettersson y Zillinger, 2011). Por tanto, es lógico que los destinos quieran conocer la secuencia de visita de los turistas, ya que este conocimiento permitirá ofrecer servicios que se adapten más a sus necesidades y mejoren su experiencia.

Analizar el comportamiento del turista es fundamental para los destinos a la hora de realizar una planificación sostenible de las regiones turísticas. Este conocimiento va a permitir identificar las zonas con más afluencia turística y realizar una mejor gestión de los flujos turísticos, algo fundamental para los operadores a la hora de ubicar sus servicios y de organizar las visitas (Xia et al., 2010). Así, este tipo de estudios tienen una aplicación directa en la creación de nuevos servicios en zonas potenciales, en la ubicación de nuevas atracciones en aquellos puntos con más flujo turístico, en el desplazamiento de ciertos servicios a zonas menos transitadas para descongestionar aquellas con mucha afluencia, en la mejora de las conexiones entre atracciones turísticas o en la interacción entre operadores turísticos, lo que mejora la capacidad turística del destino (Van del Knaap, 1999) y ayuda a prevenir aglomeraciones (Forer y Pearce, 1984; Pearce, 1987), optimizando las secuencias de visita entre los principales nodos turísticos (Forer y Pearce, 1984). Esta gestión más sostenible del destino permite aliviar algunos de los problemas que suelen tener los destinos turísticos culturales con alta densidad de turistas, como pueden ser problemas de tráfico y estacionamiento, problemas de saturación y hacinamiento (Andereck et al., 2005; McCool y Martin, 1994) o problemas de degradación de elementos naturales y patrimoniales (Andereck, 1995).

Llegados a este punto es necesario plantearnos qué variables son las que tienen influencia en el comportamiento espacio-temporal del turista. Los turistas extranjeros viajan a España con motivaciones que pueden ser bastante diferentes de las que tiene un turista nacional, incluso es posible que un turista que visita por primera vez un destino tenga una motivación diferente a otro turista que ya ha estado en otras ocasiones en el mismo lugar, lo que les llevará sin ninguna duda a tener comportamientos diferentes (Kozak, 2001; McKercher, 1998).

Hasta no hace muchos años, los investigadores interesados en estudiar el comportamiento espacio temporal de los visitantes de un determinado destino turístico no eran muy abundantes, debido 
principalmente a la dificultad que suponía conocer con precisión los lugares visitados por el turista o el tiempo que pasaba en cada uno de ellos durante toda su visita. Gracias al desarrollo de las nuevas tecnologías, concretamente del GPS, estas tareas se vuelven mucho más asequibles y precisas para los investigadores, lo que ha supuesto un mayor interés en este tipo de estudios (Neutens et al., 2011; Shoval y Isaacson, 2006).

El objetivo principal que nos planteamos es conocer la secuencia de visita que siguen los turistas cuando visitan un destino turístico y analizar cómo variables relacionadas con el lugar de origen del turista o el número de visitas previas a ese mismo destino tiene influencia en este comportamiento.

Para conocer la secuencia o ruta seguida por los turistas se ha hecho uso de la herramienta "Cronología" de Google, ya que como se verá más adelante permite monitorizar y almacenar la ubicación del turista durante toda su estancia. Para el análisis de las secuencias seguidas se usa una metodología de análisis de datos que permita trabajar con datos espacio-temporales en turismo. En particular, se utiliza la adaptación que para ciencias sociales se ha realizado de la técnica de Alineamiento de Secuencias empleando el software Clustal TXY (2.0). Como destino se ha elegido Granada. Este destino atrae a turistas de todas las nacionalidades, rozando los 3 millones de visitantes (Exceltur, 2018). Además, se elige un destino de turismo cultural puesto que además de ser una tipología de turismo con alto potencial de crecimiento en cuanto a número de turistas en España, tiene especial adecuación para la generación de experiencias de turismo.

\section{REVISIÓN DE LA LITERATURA}

\subsection{Secuencia de visita}

Los movimientos se pueden definir como el acto que tiene como resultado un cambio de lugar, de posición o de postura (Merriam-Webster, 2004). Los movimientos son un proceso dinámico que además de caracterizarse por elementos espaciales también lo hace por elementos temporales, siendo un fenómeno continuo en la línea del tiempo (Worboys y Duckham, 2004).

Así, los movimientos turísticos son los cambios espaciales que realizan los turistas dentro del lugar de actividad (Lau y McKercher, 2006). Dentro de los movimientos turísticos hay que diferenciar los movimientos intra-destino, que son los que se producen cuando los turistas se mueven dentro de un destino concreto, de los movimientos inter-destino, los que se generan cuando el turista va de un destino a otro (Leiper, 1979). Este último tipo de movimientos tradicionalmente ha sido muy trabajado en la literatura turística (Mings y McHugh, 1992; Lue et al., 1993; Oppermann, 1995; Lew y McKercher, 2002), mientras que los primeros han tenido menos atención (McKercher y Lew, 2004; Arrowsmith y Chhetri, 2003).

El estudio de estos patrones de movimiento indica cómo los turistas combinan las diferentes atracciones y cómo organizan sus horarios. Este conocimiento es de gran utilidad para los gestores de los destinos, ya que les ayuda a desarrollar paquetes turísticos, a decidir cuánto tiempo se debe abrir una atracción o a organizar el programa de actividades diarias (Xia et al., 2010).

Por otra parte, la secuencia de movimiento se puede definir como una secuencia de posiciones o de atracciones visitadas, donde se tiene en cuenta la hora de llegada y la duración de la estancia en cada una de las atracciones (Xia et al., 2005).

Para identificar la secuencia de visita de los turistas, el primer paso será conocer cuáles son las variables fundamentales que la describen. Hägerstraand (1970) propone tres tipos de restricciones cuyo efecto combinado se refleja tanto en la capacidad del individuo, como en la secuencia de las actividades que realiza en su día a día, estas tres restricciones son: capacidad, acoplamiento y limitaciones de autoridad. Para el ámbito turístico Shoval (2012) realizó una adaptación de estas restricciones, donde 
la capacidad hace referencia a las necesidades que tiene el turista de dormir, alimentarse, moverse de un lugar a otro y a la duración prevista de su estancia; el acoplamiento se refiere a la composición del grupo con el que viaja y, por último, las limitaciones de autoridad se refieren a la existencia de horarios en los transportes o en la apertura de las atracciones turísticas, entre otras.

Siguiendo a Van der Knaap (1997), distinguiremos tres ámbitos importantes a la hora de evaluar la secuencia de visita, o lo que es lo mismo, el comportamiento espacio-temporal de los turistas. Estos ámbitos son: espacio, tiempo y contexto. Por tanto, las variables que definen la secuencia de visita son tiempo y espacio, las cuales se ven condicionadas por las características (contexto) del turista (Xia y Arrowsmith, 2005). Una característica esencial para explicar dicha secuencia es la cercanía o lejanía del lugar en el que el turista reside habitualmente con el destino que visita (McKercher, 1998). Por otro lado, la experiencia previa del turista con el destino es una característica esencial a tener en cuenta para explicar la secuencia espacio-temporal de visita a un destino (Lau y MacKercher, 2006). Aunque podrían identificarse otras variables sociodemográficas y psicográficas como condicionantes de la visita, el origen del turista y su experiencia previa con el destino son características que facilitan la implementación de una comunicación y promoción de destino diferencial previa a la estancia, bien por mercado emisor o bien por contacto directo (permission marketing) con turistas que ya han estado en el destino.

\subsection{Influencia del lugar de origen del turista en la secuencia de visita}

Un elemento importante a tener en cuenta a la hora de analizar la secuencia de visita es el lugar de procedencia o de residencia del turista, que influye en los patrones de movimiento que realizará durante la visita. Por este motivo, han sido varios los autores que han realizado estudios que se basan en analizar cómo la distancia existente entre el lugar de residencia del turista y el destino elegido para pasar las vacaciones influye sobre el comportamiento que tienen los turistas en el destino y sobre su participación en las actividades organizadas (Lue et al., 1993; Oppermann, 1995; Sriram et al., 2001). Parece que cuanto mayor sea la distancia desde el país de origen, menor será la participación en este tipo de patrón de viaje.

Así mismo, se ha demostrado que el origen del turista podría tener un efecto significativo en el comportamiento de éste (Choi y Chu, 2000; Kozak, 2001; Morrison et al., 1994), ya que las fronteras son un factor que influye en la movilidad de los turistas, lo que afecta a su comportamiento en el destino (Timothy, 2002; Hall, 2005). En esta misma línea, Morrison et al. (1994) encontraron una relación entre el número de atractivos turísticos visitados y el origen del turista.

Dentro de los estudios que demuestran que muchas de estas diferencias se deben a la nacionalidad del turista, encontramos trabajos que afirman que además de la nacionalidad se deben tener en cuenta otras variables culturales que diferencian a los habitantes de un país concreto y que hace que su comportamiento se vea afectado, por ejemplo, el idioma, la religión o las experiencias previas (Farahani y Mohamed, 2013).

Otros autores, sin embargo, opinan que el origen del turista no siempre tiene que afectar al comportamiento que tiene éste durante el viaje (Flognfeldt, 1999), no quedando del todo claro el papel que juega el origen a la hora de definir la secuencia de visita.

El presente trabajo tratará de arrojar una evidencia adicional a la controversia puesta de manifiesto en la literatura sobre influencia del origen (doméstico vs internacional) en la secuencia de visita al destino.

C1: ¿Existirán diferencias en la secuencia de visita al destino cultural atendiendo al origen del turista? 


\subsection{Influencia de las visitas previas en la secuencia de visita}

La información previa que tenga el turista sobre el destino será también otro elemento que condicionará los movimientos del turista. Esta información puede proceder de varias vías, dentro de las cuales tradicionalmente han destacado los diferentes intermediarios turísticos que dan información, las guías turísticas, los comentarios de familiares y amigos, los medios sociales, etc. Este tipo de información es fundamental tanto a la hora de decidir qué atracciones visitar como en la elección de la ruta a seguir (Seaton y Bennett, 1996; Zillinger, 2006). A pesar de ello, la fuente de información más completa para un turista es su propia experiencia en el destino (Fodness y Murray, 1997 y Stewart y Vogt, 1999).

Son varios los trabajos en la literatura que han afirmado que la experiencia previa que tengan los turistas en un destino, es decir, si lo han visitado en alguna ocasión anterior, condiciona la secuencia de visita. Así, para Flognfeldt (1999), la dispersión de los movimientos va disminuyendo a medida que una persona ha visitado un mayor número de ocasiones el destino. Para McKercher (1998), el hecho de que los turistas hayan visitado anteriormente el destino, hace que elijan con más conciencia los lugares que quieren, o no, visitar evitando volver a aquellos lugares que consideran masificados y limitando su visita a un número menor de atracciones (McKercher et al., 2012). Este menor número de lugares visitados hace que los turistas de repetición se concentren solo en visitar aquellos lugares que más les atraen y puedan explorarlos con más profundidad (Oppermann, 1997).

También hay trabajos que afirman que aunque los turistas primerizos y los turistas de repetición elijan visitar los mismos lugares, el orden en el que realizan la visita puede variar, ya que la experiencia previa les ayuda a realizar una mejor planificación de su visita (Kemperman y Joh, 2003). Así, con respecto al tiempo de estancia en cada atracción turística, McKercher et al. (2012) afirman que los turistas de repetición realizan una distribución de su tiempo diferente a la que hacen los turistas primerizos. En resumen, Lehto et al. (2004) afirman que la experiencia previa influye también en la participación del turista en las actividades que se desarrollan en el destino.

C2: ¿Existirán diferencias en la secuencia de visita a Ganada atendiendo a la experiencia previa del turista con el destino?

\section{METODOLOGÍA}

\subsection{Muestra}

El primer objetivo al que nos enfrentamos antes de abordar el trabajo de campo es encontrar una herramienta que nos permita tener localizado al turista durante toda su estancia en la ciudad, para así poder conocer la ruta seguida durante su visita. Tras analizar diferentes alternativas se decidió emplear una herramienta de Google, la "Cronología" o historial de ubicaciones.

Esta herramienta comenzó a funcionar a principios del año 2012, incluida dentro de los servicios de mapas que el gigante tecnológico nos ofrece. Esta herramienta permite almacenar todos los lugares visitados y las rutas seguidas en cada uno de los desplazamientos, lo que la convierte en una herramienta ideal a la hora de analizar los patrones de comportamiento de los turistas.

El funcionamiento de esta herramienta es sencillo, operando en segundo plano, por lo que no necesita de la intervención del usuario. Simplemente necesitamos tener un dispositivo móvil asociado a una cuenta de Gmail. La red móvil y las redes WIFI trasmiten una señal geoestacionaria que le va a permitir a Google tener siempre localizado el terminal de forma más o menos precisa incluso cuando el GPS del teléfono está apagado, aunque si queremos incrementar esa precisión será necesario activarlo. El uso de esta herramienta obliga a adquirir diferentes dispositivos móviles que deben ser entregados al turista y que éste debe llevar consigo durante toda su estancia en el destino. 
Otro punto clave dentro del diseño del trabajo de campo es decidir dónde y de qué forma se captarían a los turistas. El objetivo es que esta decisión no condicione los resultados posteriores, ya que la localización inicial del turista podría tener influencia en el resto de la secuencia de visita, por lo que los turistas fueron captados en cuatro hoteles de dos, tres y cuatro estrellas ubicados en el centro de la ciudad dentro de un radio de no más de 150 metros y con una valoración similar en TripAdvisor (4 sobre 5). Además, la colaboración de los hoteles facilita disponer de un punto para la recogida de terminales y encuesta post-visita. Los hoteles concretos son Meliá Granada, Navas, Nieves y Tilos.

La muestra final está compuesta por las secuencias de visita de 303 turistas que visitaron la ciudad de Granada entre marzo y julio de 2018. La siguiente tabla muestra el porcentaje de turistas de la muestra que pertenecen a cada una de las categorías de las distintas variables sociodemográficas:

TABLA 1

Frecuencia de turistas en función de las variables sociodemográficas

\begin{tabular}{|c|c|c|}
\hline Variable & Categoría & $\%$ de turistas \\
\hline \multirow{2}{*}{ Género } & Hombre & 38,4 \\
\hline & Mujer & 61,6 \\
\hline \multirow{5}{*}{ Edad } & Menos de 25 años & 5,6 \\
\hline & De 25 a 35 años & 23,2 \\
\hline & De 36 a 50 años & 34,8 \\
\hline & De 51 a 65 años & 31,8 \\
\hline & Más de 65 años & 4,6 \\
\hline \multirow{5}{*}{ Grupo de viaje } & Solo & 5,6 \\
\hline & En pareja & 49,2 \\
\hline & En familia con niños & 16,5 \\
\hline & En familia sin niños & 12,5 \\
\hline & Con amigos & 16,2 \\
\hline \multirow{9}{*}{ Renta } & Menos de $600 €$ & 1,8 \\
\hline & De $600 €$ a $1200 €$ & 8,5 \\
\hline & De $1201 €$ a $1800 €$ & 19,8 \\
\hline & De $1801 €$ a $2400 €$ & 20,1 \\
\hline & De $2401 €$ a $3000 €$ & 15,9 \\
\hline & De $3001 €$ a $4500 €$ & 18,7 \\
\hline & De $4501 €$ a $6000 €$ & 7,1 \\
\hline & De $6001 €$ a $8000 €$ & 3,5 \\
\hline & Más de $8000 €$ & 4,6 \\
\hline
\end{tabular}

De acuerdo con el informe "Turismo de Ciudad en Andalucía", para el año 2017 un 47\% del turismo en la ciudad de Granada es doméstico, siendo idéntico el porcentaje de turistas que tienen experiencia previa con el destino (SAETA, 2017). Nuestra muestra objeto de estudio se divide en:

59 turistas nacionales que visitan la ciudad por primera vez.

120 turistas nacionales que ya habían visitado la ciudad en alguna otra ocasión.

90 turistas extranjeros que visitan la ciudad por primera vez.

26 turistas extranjeros que ya habían visitado la ciudad en alguna otra ocasión.

Los turistas nacionales provenían de todas las comunidades autónomas españolas, mientras que los turistas extranjeros vienen de 34 países diferentes pertenecientes a los 5 continentes. 
El perfil del turista nacional es de una persona de 44 años, casada y con hijos que tiene estudios superiores y que está en la ciudad durante 38 horas. Por su parte, el perfil del turista internacional es de una persona de 46 años también casada, con hijos y con estudios universitarios que pasa en la ciudad casi 41 horas.

\subsection{Preparación y contextualización de los datos}

El objetivo de esta fase es depurar los datos y prepararlos para su análisis, ya que no es factible trabajar con los datos de localizaciones que directamente proporciona la Cronología de Google (latitud, longitud y tiempo, medidas a intervalos de cinco minutos). Sería imposible encontrar secuencias similares en ubicaciones con tal grado de detalle.

El primer paso será dividir el mapa del destino en diferentes zonas o regiones, asignando a cada una de ellas una letra que será la que identifique las regiones que ha visitado el turista. En este caso la ciudad ha sido dividida en 10 polígonos o zonas geográficas (identificadas con letras de la A a la J) para así poder asignar cada posición GPS a cada una de estas zonas. Esta división sigue la distribución en distritos de la ciudad de Granada (figura 1).

FIGURA 1

División de la ciudad en distritos

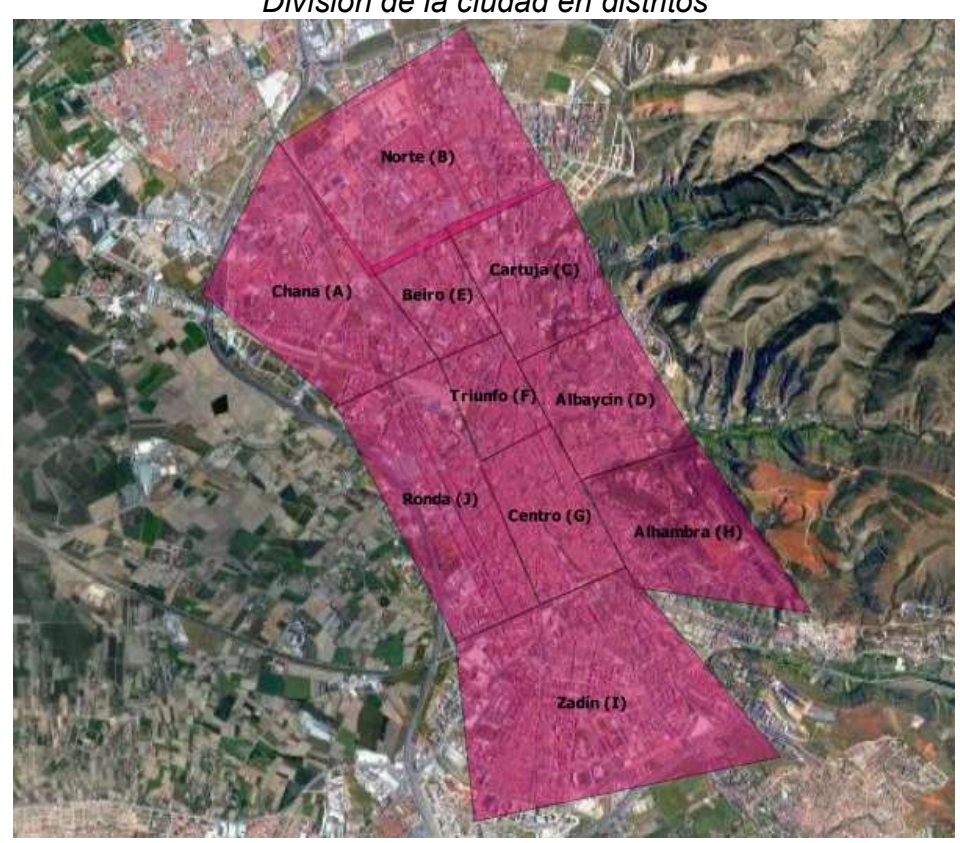

A continuación, se asigna a cada turista la secuencia de zonas visitadas, la cual se corresponde con una secuencia de letras que identifican a cada uno de los diferentes lugares de la ciudad visitados, teniendo siempre en cuenta el orden de visita. De esta forma se condensa el conjunto de posiciones GPS de un turista en una secuencia espacio-temporal de visita.

En este punto se tendrá también en cuenta el tiempo que el turista pasa en cada una de las zonas, codificado la duración de la visita con nuevos caracteres en función de si el turista pasa entre 30 y 90 minutos (x), entre 91 y 150 minutos (y) o más de 150 minutos (z) visitando una misma zona. Este carácter se ha colocado tras la letra que identifica la zona visitada. Así, un ejemplo de secuencia obtenida sería:

\section{DyGzHxDz}

Donde las letras mayúsculas identifican a la zona visitada y las letras minúsculas definen el tiempo que dedica a la visita de la misma. 
Para evitar que los desplazamientos enturbien la secuencia de visita, se han eliminado del análisis aquellas zonas donde el turista ha pasado menos de 30 minutos ya que, según las dimensiones de las mismas, es un tiempo tan limitado que no permite que el turística pueda llegar a explorarlas, considerándose, por tanto, zonas de paso que no afectarán a la secuencia de visita. La tabla 2 incluye el resumen de códigos utilizados para recoger la información de localizaciones de cada turista.

\subsection{Alineamiento de secuencias}

El alineamiento de secuencias es una metodología propia de la investigación en bioquímica desarrollada por Sankoff y Kruskal (1983) para analizar secuencias de proteínas y ADN. Años más tardes, investigadores de otras disciplinas se dieron cuenta de que la aplicación de esta metodología a sus ámbitos de estudio podría arrojar resultados interesantes, teniendo desde entonces aplicación en estudios de sociología, economía o marketing (Halpin y Chan, 1998; Blair-Loy, 1999; Stovel y Bolan, 2004).

Esta metodología se basa en la comparación de los caracteres que forman las cadenas usando unas medidas de similitud y de distancia. Para obtener estas medidas se va comparando uno a uno cada carácter, haciendo transformaciones hasta conseguir que las cadenas sean lo más parecidas posibles. Para ello usan tres tipos de transformaciones: la inserción de un carácter en una de las posiciones, el borrado o eliminación de un carácter de una de las posiciones y la sustitución (combinar un borrado y una inserción de forma que se sustituya el carácter de una posición por el equivalente al que hay en esa misma posición de la otra cadena). Cada operación va restando o sumando una puntuación a esa medida de similitud o distancia, repitiendo el proceso hasta encontrar aquel alineamiento que minimice la distancia entre las cadenas alineadas (Shoval e Isaacson, 2007; Wilson, 1998).

Este proceso nos va a permitir identificar aquellos caracteres más habituales dentro de un conjunto de cadenas de secuencias, lo que aplicado a nuestro estudio nos ofrecerá la ruta típica que sigue cada conjunto de turistas que visitan la ciudad, teniendo en cuenta no solamente las zonas visitadas, sino también el orden en el que se visitan y el tiempo en el que se permanece en ellas.

Para su análisis se hizo uso del software Clustal TXY (2.0) (Wilson, 2016), adaptación del tradicional software Clustal X usado de forma habitual en biología (Thompson et al., 1994). Esta nueva versión del software fue creada para facilitar las investigaciones en Ciencias Sociales.

\section{RESULTADOS}

El primer paso necesario para identificar la "secuencia consenso" o ruta típica seguida por los turistas es alinear por separado mediante un alineamiento múltiple todas las secuencias de visita de cada uno de nuestros segmentos: turistas nacionales que visitan la ciudad por primera vez, turistas nacionales que ya habían visitado la ciudad en alguna otra ocasión, turistas extranjeros que visitan la ciudad por primera vez y turistas extranjeros que ya habían visitado la ciudad en alguna otra ocasión.

A continuación, hay que realizar dos alineamientos de perfil, uno donde se introduzcan las secuencias alineadas de cada grupo de turistas nacionales y otro donde los datos introducidos sean las secuencias alineadas de cada grupo de turistas extranjeros. Esto nos permitirá comparar por un lado las rutas de los turistas nacionales que visitan por primera vez el destino frente a las rutas de aquellos turistas nacionales que ya lo habían visitado previamente y, por otro lado, las rutas de los turistas extranjeros que visitan por primera vez el destino frente a las rutas de aquellos turistas extranjeros que ya lo habían visitado previamente. Como ejemplo, en el anexo se muestra una imagen del output que ofrece Clustal TXY tras realizar el alineamiento de perfil y calcular la secuencia consenso. 
TABLA 2

Identificador de distritos y calificador de duración de la visita

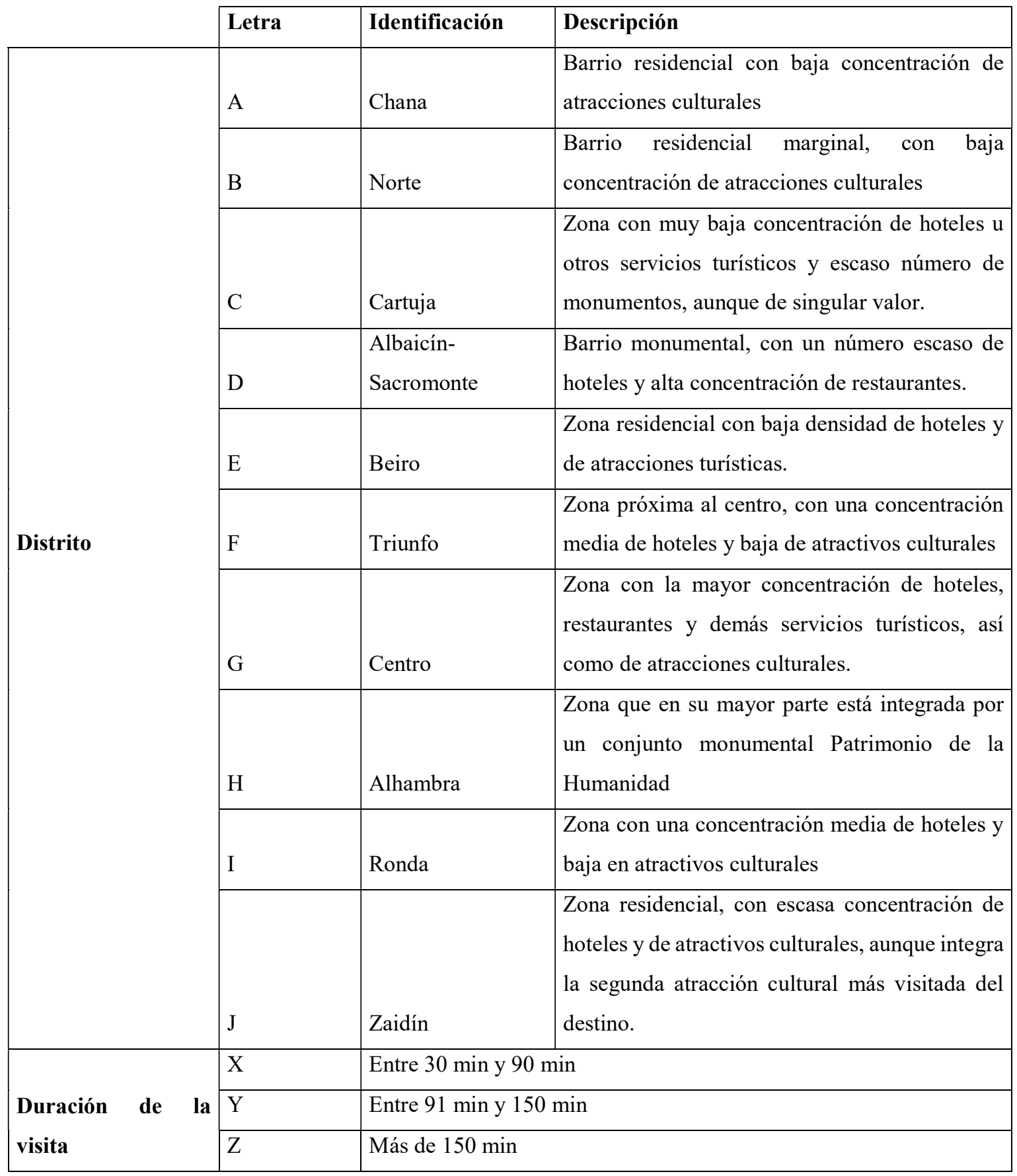

Fuente: Elaboración propia.

El resultado serán cuatro secuencias consenso que corresponden a las rutas típicas seguidas por cada uno de los cuatro segmentos de turistas, siendo las siguientes:

- Turistas nacionales que visitan la ciudad por primera vez, su secuencia típica es "GzHxDx" (figura 2). Estos turistas dedican la mayor parte de su estancia, más de 2,5 horas, a pasear por el centro de la ciudad (letra 'G'). Posteriormente visitan la Alhambra (H) y el barrio del Albaicín (D), a cada una de estas zonas dedican entre 90 y 150 minutos. Estos turistas realizan una ruta que podría considerarse como la ruta "típica" para cualquier turista que no conozca la ciudad, sin duda visitan las tres principales zonas turísticas de la ciudad. 


\section{FIGURA 2}

Mapas de calor que representan los lugares más visitados por los turistas nacionales que visitan la ciudad por primera vez

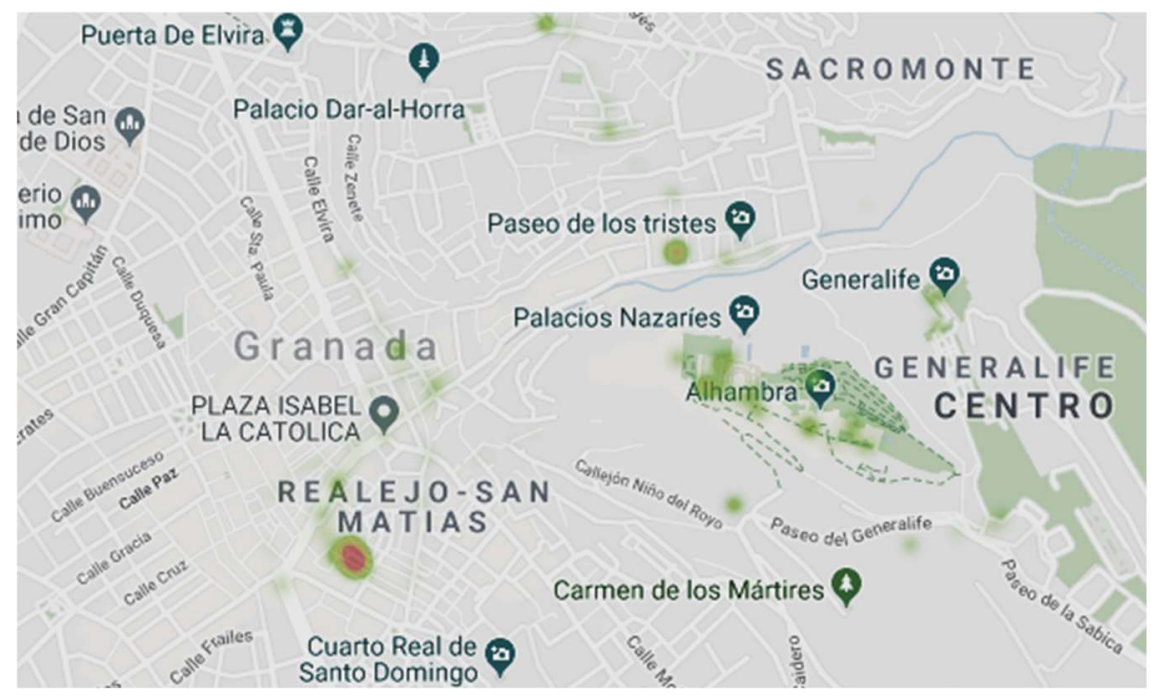

- Turistas nacionales que ya habían visitado la ciudad en alguna otra ocasión, la secuencia consenso nos indica que la ruta típica de este tipo de turistas es "Gz" (figura 3). Esto nos indica que los turistas españoles que ya habían estado previamente en la ciudad dedican prácticamente toda su estancia en la ciudad a pasear por el centro de la misma, a pesar de ello, esto no significa que sea la única zona visita, sino que son turistas que pueden visitar otras zonas pero no hay ninguna zona con un volumen de visitas tan significativo como para pertenecer a la ruta consenso de este segmento.

\section{FIGURA 3}

Mapas de calor que representan los lugares más visitados por los turistas nacionales que anteriormente ya habían visitado la ciudad

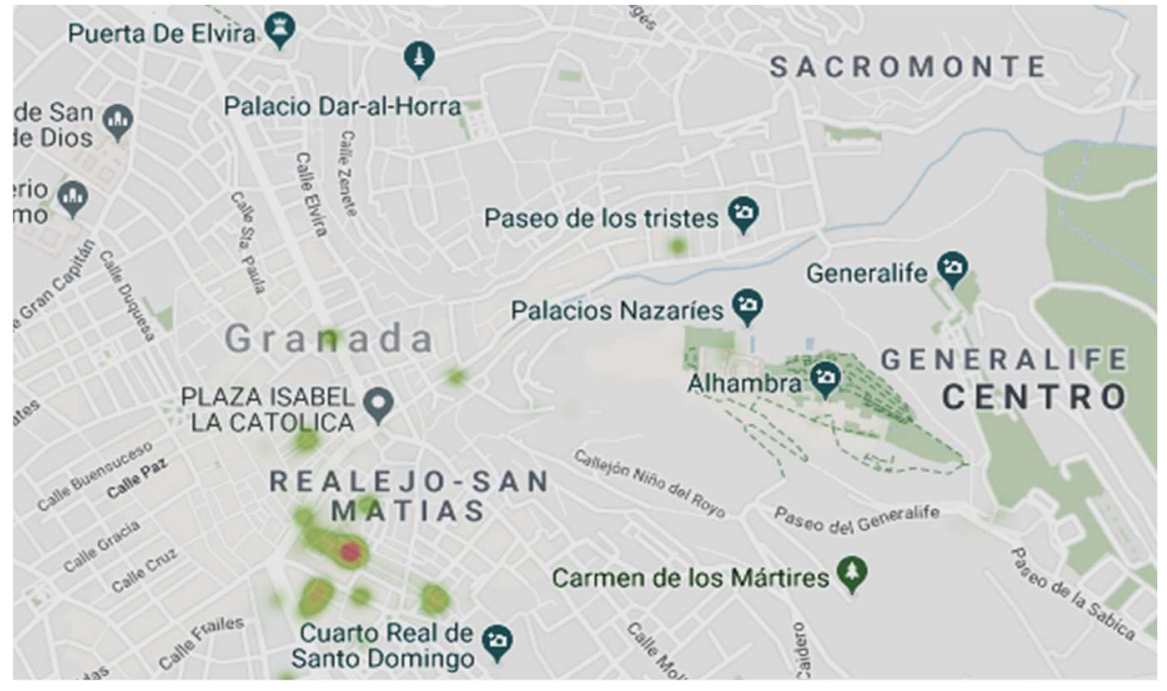

- Turistas extranjeros que visitan la ciudad por primera vez, para este grupo de turistas obtenemos como secuencia consenso "GzHxGz" (figura 4). Esta secuencia consenso nos indica que este grupo de turista dedican mucho tiempo a visitar las atracciones turísticas del centro de la ciudad (G), esta zona es visitada en dos ocasiones, dedicando en cada una de ellas más de 150 minutos. Además, este grupo de turistas también visita la Alhambra dedicando entre 1.5 a 2.5 horas. 


\section{FIGURA 4}

Mapas de calor que representan los lugares más visitados por los turistas extranjeros que visitan la ciudad por primera vez

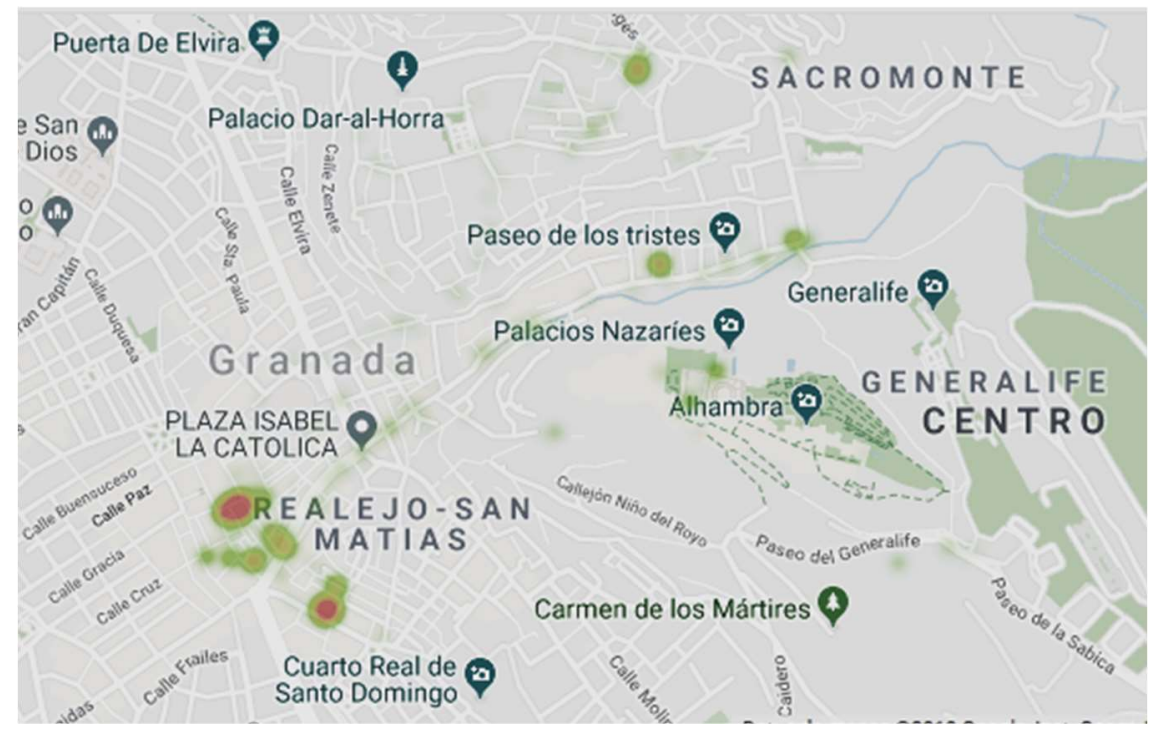

- Turistas extranjeros que ya habían visitado la ciudad en alguna otra ocasión, la secuencia consenso para este grupo es "GzGzGzHx" (figura 5). El hecho de que la zona "G" aparezca en tres ocasiones nos indica que son turistas que dedican gran parte de su estancia a visitar el centro de la ciudad, además no se olvidan de realizar una visita al Conjunto Monumental de La Alhambra y el Generalife, aunque le dedican menos de 2.5 horas a esta visita.

\section{FIGURA 5}

Mapas de calor que representan los lugares más visitados por los turistas extranjeros que anteriormente ya habían visitado la ciudad

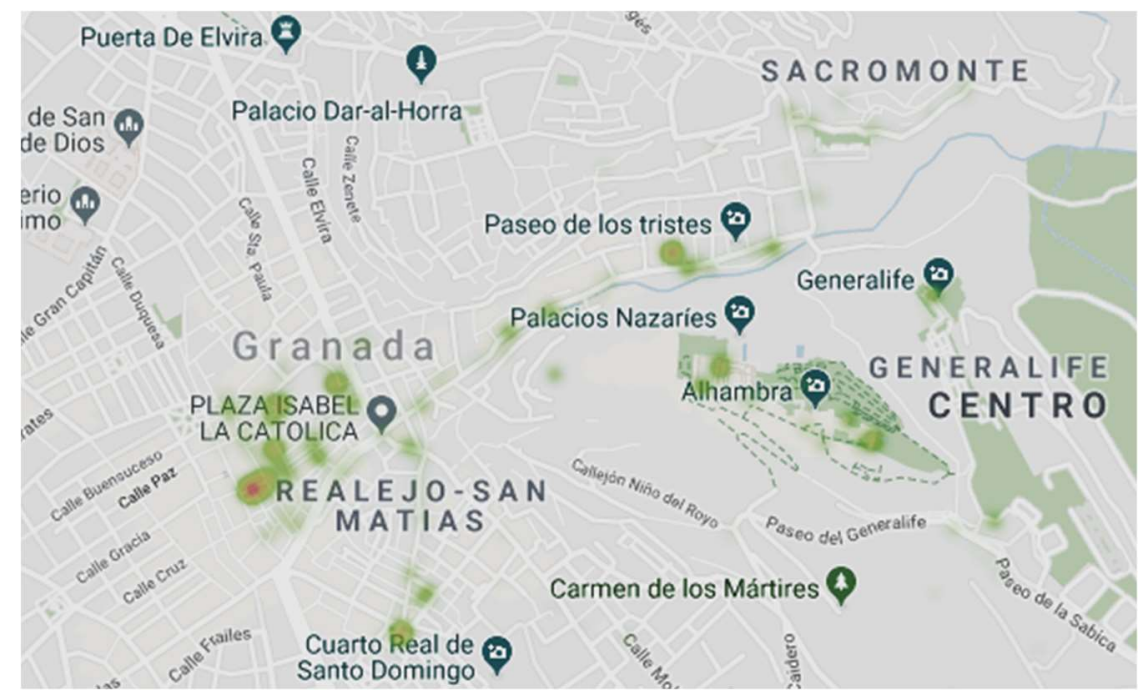

Conjuntamente, los resultados obtenidos permiten responder afirmativamente a las dos cuestiones de investigación planteadas, sugiriendo que tanto el origen del turista como el conocimiento previo del destino tienen influencia en la secuencia espacio-temporal de su visita. 


\section{DISCUSIÓN DE RESULTADOS.}

A la hora de analizar este comportamiento espacio-temporal o secuencia de visita, es imprescindible conocer las variables que tiene influencia en el mismo, para así poder enfocar los esfuerzos y ofrecer servicios diferentes a cada segmento de turistas en función de sus necesidades. Estudios previos han demostrado que variables como el lugar de procedencia del turista o el número de visitas previas (experiencia anterior con el destino) va a determinar en gran medida esa ruta o secuencia de visita (Choi y Chu, 2000; Flognfeldt, 1999; Kozak, 2001; McKercher, 1998; Morrison et al., 1994).

Como era de esperar, en la secuencia típica de cada uno de los cuatro segmentos considerados están las tres principales zonas turísticas de Granada. Es más, solamente la zona centro es común en los cuatro segmentos, dedicando en todos los casos la mayor parte de su tiempo de estancia a estar en dicha zona. Por tanto, se constata que el problema de la saturación del turismo en Granada va más allá del cociente entre visitantes por periodo y residentes (indicador conocido como presión turística de un destino), dado que muchos de estos turistas concentran gran parte de su visita en un distrito.

Por otro lado, parece lógico pensar que el Conjunto Monumental de La Alhambra y Generalife constituyen el atractivo principal del destino con 2.5 millones de entradas vendidas en 2016. De acuerdo con la teoría de nodos de Dredge (1999), un turista visitará los nodos principales del destino que motivaron su visita y, de manera accesoria, se planteará visitas otros atractivos del destino si el tiempo y características de la estancia se lo permiten. Lo que destaca en los resultados de nuestra investigación es que hay un segmento de turistas para el que tras su primera visita el que considera nodo principal cambia a la zona centro (turistas nacional que repiten su visita).

Finalmente, y respecto al orden en el que se produce la visita al Conjunto Monumental de La Alhambra y Generalife, los resultados obtenidos están en consonancia con la literatura sobre la distribución de los movimientos del turista durante la estancia, al menos para el turismo doméstico e internacional de primera visita. En los primeros días de la visita el turista tiende a caminar más, permaneciendo más estático en los últimos momentos de su visita (Zillinger, 2007; Kemperman et al., 2009), debido a que los primeros días el turista querrá ver aquello por lo que ha venido, visitar lugares de interés turístico y explorar la ciudad (Lee et al., 2015). Los primeros visitantes planifican su estancia para visitar La Alhambra en mitad de su ruta típica. Sin embargo, para los turistas de repetición en el caso de los domésticos ya se ha comentado que este monumento no forma parte de su ruta de consenso y para los internacionales ocupa la última posición en dicha ruta. Este resultado hace pensar que para los turistas que repiten la motivación principal de visita puede haber cambiado, considerando el centro de la ciudad como punto principal de interés turístico.

En resumen, los resultados obtenidos son coherentes con la literatura sobre movimientos de los turistas en el destino, aunque sugieren como hecho novedoso que para turistas que repiten puede producirse una permuta en cuanto a nodo principal de atracción. Es posible que el nodo que atrae la primera visita no sea el único que contribuye a la retención del turista. De ahí la importancia de la complementariedad de atractivos en un destino (Benur y Bramwell, 2015).

Cabe también destacar la implicación que este trabajo tiene en la gestión turística del destino. Tal y como indica la literatura previa, conocer estos resultados va a permitir mejora la capacidad turística del destino (Van del Knaap, 1999), ayudar a prevenir aglomeraciones (Forer y Pearce, 1984; Pearce, 1987) y optimizar las secuencias de visita entre los principales nodos turísticos (Forer y Pearce, 1984).

\section{CONCLUSIONES}

Tal y como se ha comentado, analizar el comportamiento espacio-temporal que siguen los turistas cuando visitan un destino resulta fundamental para ayudarle a obtener una experiencia de visita 
agradable que le lleve a tener un comportamiento de lealtad hacia el destino y haga aumentar los beneficios del lugar (Geva y Goldman, 1991; Knutson y Beck, 2004).

En el caso que nos ocupa, un alineamiento de secuencias nos ha ofrecido resultados sobre las rutas típicas seguidas por cuatro segmentos diferentes de turistas: nacionales que visitan Granada por primera vez, nacionales que ya habían visitado el destino en alguna otra ocasión, extranjeros que viajan a la ciudad por primera vez y extranjeros que vuelven al destino.

Una de las principales conclusiones que podemos obtener de este análisis es que estos segmentos concentran su actividad en las principales zonas turísticas del destino: centro, Albaicín-Sacromonte y el Conjunto Monumental de la Alhambra y el Generalife. La implicación inmediata es un aumento de la presión turística en estas zonas muy por encima del dato de turistas entre residentes.

Con respecto a las diferencias entre los grupos, destaca que para los turistas extranjeros la visita a La Alhambra es obligada aunque ya hayan viajado a la ciudad en otras ocasiones. El hecho de que sean turistas que tienen que recorrer más distancia para llegar, hace que decidan visitar siempre el principal monumento de la ciudad. Sin embargo, parece que los turistas que repiten su visita planifican ésta considerando otros atractivos de la ciudad, complementarios al conjunto monumental de La Alhambra y Generalife.

Por otro lado, la visita que realizan a este conjunto monumental tiene una duración escasa, menor a 2.5 horas en todos los segmentos, cuando la extensión del mismo y la riqueza de sus atractivos sugieren que este tiempo debe ser considerablemente superior.

Del conjunto de estas conclusiones surge una recomendación para destinos con una elevada saturación, como es el caso de Granada. Es fundamental promover la complementariedad de atracciones y olvidar el monoproducto, por muy valioso que sea la atracción principal del destino. Mientras que la atracción principal de Granada contribuye de forma decisiva a la atracción del turista por primera vez, lo que hace que este vuelva al destino es la complementariedad de este conjunto monumental con otras zonas turísticas de la ciudad. En este sentido, esta complementariedad debería ir más allá de la zona centro o de los barrios del Albaicín y Sacromonte, mejorando la derivación de turistas hacia atracciones como son el Parque de las Ciencias, Plaza de Toros o Cartuja, donde se cuenta con atracciones culturales de singular valor, algunas de ellas declaradas BIC o Patrimonio Histórico Andaluz.

Dentro de las limitaciones cabe destacar la dificultad del trabajo de campo. El hecho de tener que monitorizar todos los movimientos de los turistas hace que sea un trabajo complejo y que en muchos casos crea rechazo en los turistas, negándose a participar en la investigación. Además, por la duración limitada de la batería del dispositivo, no podemos tener recorridos superiores a 72 horas. Estos hechos suponen una restricción a la hora de seleccionar a la muestra, haciendo que alguno de los segmentos este formado por un número bajo de turistas, lo que obliga a analizar los resultados con cautela.

Este trabajo se desarrolla en la ciudad de Granada, lo que supone una limitación a la hora de extrapolar las conclusiones a otros lugares. Por ello, como futura línea de investigación planteamos la réplica del estudio en otras localizaciones.

Otra de las futuras líneas de investigación que se plantean es analizar más en profundidad a qué se deben esos cambios en el comportamiento relacionados con el lugar de origen del turista y su experiencia previa con el destino. Quizá sea bueno tener en cuenta variables relacionadas con la distancia psicológica que nos pueda ayudar a entender mejor estas diferencias. 


\section{REFERENCIAS BIBLIOGRÁFICAS}

ANDERECK, K.L., (1995). Environmental consequences of tourism: a review of recent research. Environmental Consequences of Tourism: A Review of Recent Research, (INT-323), 77-81.

ANDERECK, K. L., VALENTINE, K. M., KNOPF, R. C., \& VOGT, C. A. (2005). Residents' perceptions of community tourism impacts. Annals of tourism research, 32(4), 1056-1076.

ARROWSMITH, C., \& CHHETRI, P. (2003). Port Campbell National Park: Patterns of Use. A Report for the Development of a Visitor Typology as Input to a Generic Model of Visitor Movements and Patterns of Use. Melbourne.

BENUR, A. M., \& BRAMWELL, B. (2015). Tourism product development and product diversification in destinations. Tourism Management, 50, 213-224.

BLAIR-LOY, M. (1999). Career patterns of executive women in finance: An optimal matching analysis. American Journal of Sociology, 104(5), 1346-1397.

CHOI, T. Y., \& CHU, R. (2000). Levels of satisfaction among Asian and Western travellers. International Journal of Quality \& Reliability Management, 17(2), 116-132.

DREDGE, D. (1999). Destination place planning and design. Annals of Tourism Research, 26(4), 772-791.

EXCELTUR. (2018). Balance Empresarial Del Año 2017. Disponible en: http://www.exceltur.org/wpcontent/uploads/2018/01/Informe-Perspectivas-N63-Balance-del-a\%C3\%B1o-2017-y-previsiones-para-ela\%C3\%B10-2018.pdf

FARAHANI, B. M., \& MOHAMED, B. (2013). Relationship between Nationality and Tourists Behavior; Case of Middle East Tourists in Malaysia from Tour Guides Perspective Relationship between nationality and tourists ' behaviour: case of Middle East tourists in Malaysia from tour guides perspectiv. Tourism Anthropology, 3(January), 43-57. doi:10.1504/IJTA.2013.054409

FLOGNFELDT JR, T. (1999). Traveler geographic origin and market segmentation: The multi trips destination case. Journal of Travel \& Tourism Marketing, 8(1), 111-124.

FODNESS, D., \& MURRAY, B. (1997). Tourist information search. Annals of Tourism Research, 24(3), 503-523.

FORER, P.C., \& PEARCE, D.G. (1984). Spatial patterns of package tourism in New Zealand. New Zealand Geographer, 40(1): 34-43.

Geva, A., \& Goldman, A. (1991). Satisfaction measurement in guided tours. Annals of Tourism Research, 18(2), 177-185.

HÄGERSTRAAND, T. (1970). What about people in regional science? Papers in Regional Science, 24(1), 7-24.

HALL, C. M. (2005). Reconsidering the geography of tourism and contemporary mobility. Geographical Research, 43(2), 125-139.

HALPIN, B., \& CBAN, T. W. (1998). Class careers as sequences: An optimal matching analysis of work-life histories. European Sociological Review, 14(2), 111-130.

INE (2017). Datos de turismo llegadas turísticas a España. Disponible en: http://www.ine.es/prodyser/espa_cifras/2017/index.html\#50/z

KEMPERMAN, A. D. A. M., BORGERS, A. W. J., \& TIMMERMANS, H. J. P. (2009). Tourist shopping behavior in a historic downtown area. Tourism Management, 30(2), 208-218.

KEMPERMAN, A. D. A. M., \& JOH, C.-H. (2003). Comparing first-time and repeat visitors activity patterns. Tourism Analysis, 8(2), 159-164.

KNUTSON, B. J., \& BECK, J. A. (2004). Identifying the dimensions of the experience construct: development of the model. Journal of Quality Assurance in Hospitality \& Tourism, 4(3-4), 23-35.

KOZAK, M. (2001). Repeaters' behavior at two distinct destinations. Annals of Tourism Research, 28(3), 784-807.

LAU, G., \& MCKERCHER, B. (2006). Understanding tourist movement patterns in a destination: A GIS approach. Tourism and Hospitality Research, 7(1), 39-49. doi:10.1057/palgrave.thr.6050027

LEE, S. K., JEE, W. S. F., FUNK, D. C., \& JORDAN, J. S. (2015). Analysis of attendees' expenditure patterns to recurring annual events: Examining the joint effects of repeat attendance and travel distance. Tourism Management, 46, 177-186.

LEHTO, X. Y., O'LEARY, J. T., \& MORRISON, A. M. (2004). The effect of prior experience on vacation behavior. Annals of Tourism Research, 31(4), 801-818.

LEIPER, N. (1979). The framework of tourism: Towards a definition of tourism, tourist, and the tourist industry. Annals of Tourism Research, 6(4), 390-407.

LEW, A. A., \& MCKERCHER, B. (2002). Trip destinations, gateways and itineraries : the example of Hong Kong, 23, 609-621.

LEW, A., \& MCKERCHER, B. (2006). Modeling tourist movements: a local destination analysis. Annals of Tourism Research, 33(2), 403-423. doi:10.1016/j.annals.2005.12.002

LUE, C.-C., CROMPTON, J. L., \& FESENMAIER, D. R. (1993). Conceptualization of multi-destination pleasure trips. Annals of Tourism Research, 20(2), 289-301.

MCCOOL, S. F., \& MARTIN, S. R. (1994). Community attachment and attitudes toward tourism development. Journal of Travel research, 32(3), 29-34.

MCKERCHER, B. (1998). The effect of market access on destination choice. Journal of Travel Research, 37(1), 39-47.

MCKERCHER, B., \& LEW, A. A. (2004). Tourist flows and the spatial distribution of tourists. A Companion to Tourism, 36-48.

MCKERCHER, B., SHOVAL, N., NG, E., \& BIRENBOIM, A. (2012). First and repeat visitor behaviour: GPS tracking and GIS analysis in Hong Kong. Tourism Geographies, 14(1), 147-161. 
MERRIAM-WEBSTER. (2004). Merriam-Webster's collegiate dictionary. Merriam-Webster.

MINGS, R. C., \& MCHUGH, K. E. (1992). The spatial configuration of travel to Yellowstone National Park. Journal of Travel Research, 30(4), 38-46.

MINISTERIO DE ENERGÍA, T. Y A. D. (2017). España logra un récord de llegadas en 2017 con 82 millones de turistas internacionales, 39-40. Disponible en: http://www.minetad.gob.es/esES/GabinetePrensa/NotasPrensa/2018/Paginas/Espa\%C3\%B1alograunr\%C3\%A9corddellegadasen2017con 82millonesdeturistasinternacionales.aspx

MORRISON, A. M., HSIEH, S., \& T O'LEARY, J. (1994). A comparison of the travel arrangements of international travelers from France, Germany and the UK. Tourism Management, 15(6), 451-463.

NEUTENS, T., SCHWANEN, T., \& WITLOX, F. (2011). The Prism of Everyday Life: Towards a New Research Agenda for Time Geography. Transport Reviews, 31(1), 25-47. doi:10.1080/01441647.2010.484153

OPPERMANN, M. (1995). A Model of Travel Itineraries, (1994), 57-61.

OPPERMANN, M. (1997). First-time and repeat visitors to New Zealand. Tourism Management, 18(3), $177-181$.

PEARCE, D.G. (1987). Spatial patterns of package tourism in Europe. Annals of tourism research, 14(2), 183201.

PETTERSSON, R., \& ZILLINGER, M. (2011). Time and space in event behaviour: Tracking visitors by GPS. Tourism Geographies, 13(1), 1-20.

SAETA, 2017. Turismo de Ciudad en Andalucía. Disponible en: http://www.juntadeandalucia.es/turismoydeporte/publicaciones/estadisticas/perfil_prof_ciudad_ago17.pdf.

SANKOFF, D., \& KRUSKAL, J. B. (1983). Time Warps, String Edits, and Macromolecules: The Theory and Practice of Sequence Comparison. Addison-Wesley.

SEATON, A. V, \& BENNETT, M. M. (1996). The marketing of tourism products: Concepts, issues and cases. Cengage Learning EMEA.

SHOVAL, N. (2012). Time geography and tourism. In J. Wilson (Ed.), The Routledge handbook of tourism geographies (pp. 174-180). Abingdon: Routledge

SHOVAL, N., \& ISAACSON, M. (2006). Application of tracking technologies to the study of pedestrian spatial behavior. Professional Geographer, 58(2), 172-183. doi:10.1111/j.1467-9272.2006.00524.x

SHOVAL, N., \& ISAACSON, M. (2007). Sequence Alignment as a Method for Human Activity Analysis in Space and Time. Annals of the Association of American Geographers, 97(2), 282-297. doi:10.1111/j.14678306.2007.00536.x

SRIRAM, N., LEW, A. A., \& RAGURAMAN, K. (2001). Gateways, hubs and destinations: transportation hierarchies in Southeast Asia. In Tourism management and policy: Perspectives from Singapore (pp. 55-90). World Scientific.

STEWART, S. I., \& VOGT, C. A. (1999). A case-based approach to understanding vacation planning. Leisure Sciences, 21(2), 79-95.

STOVEL, K., \& BOLAN, M. (2004). Residential trajectories: Using optimal alignment to reveal the structure of residential mobility. Sociological Methods \& Research, 32(4), 559-598.

THOMPSON, J. D., HIGGINS, D. G., \& GIBSON, T. J. (1994). Improved sensitivity of profile searches through the use of sequence weights and gap excision. Bioinformatics, 10(1), 19-29.

TIMOTHY, D. J. (2002). Tourism and political boundaries. Routledge.

VAN DER KNAAP, W. G. M. (1997). The Tourist's Drives: GIS oriented methods for analysing tourist recreation complexes. Van der Knaap.

VAN DER KNAAP, W.G.M. (1999). Research report: GIS-oriented analysis of tourist time-space patterns to support sustainable tourism development, Tourism Geographies, 1(1): 56-69. doi:10.1080/14616689908721294

WILSON, C. (2016). Notes on ClustalTXY and ClustalG A sequence alignment software package for application in social and natural sciences., (June).

WILSON, W. C. (1998). Activity pattern analysis by means of sequence-alignment methods. Environment and Planning A, 30(6), 1017-1038.

WORBOYS, M. F., \& DUCKHAM, M. (2004). GIS: a computing perspective. CRC press.

XIA, J. (CECILIA), EVANS, F. H., SPILSBURY, K., CIESIELSKI, V., ARROWSMITH, C., \& WRIGHT, G. (2010). Market segments based on the dominant movement patterns of tourists. Tourism Management, 31(4), 464469. doi:10.1016/j.tourman.2009.04.013

XIA, J., CIESIELSKI, V., \& ARROWSMITH, C. (2005). Data mining of tourists spatio-temporal movement patterns: A case study on Phillip Island. Proceedings of the Eighth ..., 1-15. Retrieved from http://www.cs.rmit.edu.au/ vc/papers/geocomputation-xia.pdf

ZILLINGER, M. (2006). The importance of guidebooks for the choice of tourist sites: A study of German tourists in Sweden. Scandinavian Journal of Hospitality and Tourism, 6(3), 229-247.

ZILLINGER, M. (2007). Tourist Routes: A Time-Geographical Approach on German Car-Tourists in Sweden. Tourism Geographies, 9(1), 64-83. doi:10.1080/14616680601092915 